\title{
Social Activity and Healthy Aging: A Study of Aging Danish Twins
}

\author{
Matt McGue' and Kaare Christensen² \\ 'Department of Psychology, University of Minnesota, Minneapolis, Minnesota, United States of America \\ ${ }^{2}$ The Danish Twin Registry and Department of Epidemiology, Institute of Public Health, Southern Denmark University, Odense, Denmark
}

\begin{abstract}
Ithough social and intellectual engagement have been consistently associated with late-life functioning, rather than true causation, these associations may reflect the experiential choices of high functioning individuals (i.e., selection effects). We investigated the association of social activity with late-life physical functioning, cognitive functioning, and depression symptomatology using data from 1112 pairs of like-sex twins who participated in the Longitudinal Study of Aging Danish Twins. Consistent with previous research, we found that social activity was significantly correlated with overall level of physical functioning, cognitive functioning, and depression symptomatology. We also found that social activity was significantly and moderately heritable (estimate of .36), raising the possibility that its association with late-life functioning might reflect selection processes. Further, social activity did not predict change in functioning and in monozygotic twin pairs discordant on level of social activity, the more socially active twin was not less susceptible to age decreases in physical and cognitive functioning and increases in depression symptomatology than the less socially active twin. These results are interpreted in the context of the additional finding that nonshared environmental factors, although apparently not social activity, are the predominant determinant of changes in late-life functioning.
\end{abstract}

The hypothesis that an active lifestyle is essential to living a happy, healthy and long life is the basis for public health recommendations (National Institute of Health [NIH], 1996), and is captured by the popular maxim, 'Use it or lose it'. Nonetheless, although an active and engaged lifestyle undoubtedly contributes to physical, psychological and cognitive health in late life, it has been difficult for researchers to precisely quantify and characterize the nature of this contribution. Several factors have contributed to the difficulty of unambiguously identifying the effect of an active lifestyle. The association between activity and functioning, rather than reflecting a direct causal influence, may owe to their common correlation with other lifestyle and medical risk factors. Individuals who are active are also likely to not smoke, not be obese, be highly educated, and have good access to health care (Rowe \& Kahn, 1999; Vaillant \& Mukamal, 2001). Statistical adjustment for confounded effects is a common, and we believe legitimate, approach to trying to isolate the effect of a variable of interest (e.g., estimating the association of physical activity and late-life health controlling statistically for the correlation of both with other lifestyle factors like smoking and weight). Nonetheless, statistical control alone cannot entirely resolve the issue of causal ambiguity since a residual association could always be accounted for by some unmeasured covariate.

A related, and we believe more significant, issue is what has been termed the 'selection problem' the possibility that the association between activity and indicators of late-life functioning reflect the lifestyle selections of genetically healthier individuals (Kujala et al., 2002, 1998). For example, individuals with an inherited disposition for high cognitive functioning may be more likely to engage in intellectual pursuits than individuals without this inherited advantage simply because their genetic constitution makes these activities easier and more enjoyable to engage in. The selection problem, which is characterized as a gene-environment correlation by behavioral geneticists, raises the possibility that the association of lifestyle factors with late-life functioning may reflect the causal influence of the latter on the former rather than the converse.

Experimental interventions are the most direct way of resolving the contribution of lifestyle factors to latelife functioning. Nonetheless, the effects of short-term but intense lifestyle changes may not adequately capture the long-term impact of lifestyle factors that play out over the course of many years rather than just several weeks. Alternatively, investigators have used prospective longitudinal studies in an attempt to resolve causal ambiguity. Because initial levels of functioning are thought to capture the impact of selection effects, an

Received 14 January, 2007; accepted 29 January, 2007.

Address for correspondence: Matt McGue, Department of Psychology/Elliott Hall, University of Minnesota, Minneapolis, MN 55455, USA. E-mail: mcgue001@umn.edu 
association between a lifestyle factor and change in functioning is considered supportive of true causation. Nonetheless, lifestyle exposure may be heritable (e.g., Frederiksen \& Christensen, 2003), so that its association with initial level or change in functioning may be genetically rather than environmentally mediated.

Finally, the co-twin control approach attempts to resolve causal ambiguity by determining whether lifestyle differences between genetically identical individuals, which must be environmental in origin, are associated with differences in their functioning. Crowe et al. (2003) provide an illustration of the use of co-twin controls to address the selection problem. Replicating previous research, these investigators found that individuals who develop Alzheimer's disease (AD) were less likely than controls to have had an active lifestyle 20 years prior to their clinical evaluation. But does the lower rate of activity seen in individuals who develop $\mathrm{AD}$ reflect a direct causal influence of leisure activity on risk of $\mathrm{AD}$, or might reduced activity be an early consequence of the cognitive deficiencies experienced by individuals who develop $\mathrm{AD}$, due perhaps to reduced cognitive reserve (Gatz et al., 2001; Mortimer, 1997)? By showing further that reduced activity differentiates cases from controls within twin pairs discordant for developing $\mathrm{AD}$, Crowe et al. (2003) were able to conclude that genetic selection effects could not account entirely for the observed association of activity with $\mathrm{AD}$.

The present study uses the co-twin control method to investigate the relationship of social activity with three domains of late-life functioning: physical functioning, cognitive functioning, and depression symptomatology. We make use of a large sample of over 1000 pairs of likesex twin participants in the Longitudinal Study of Aging Danish Twins (LSADT; Christensen et al., 1999) to address the following questions:

1. What is the nature of late-life age changes in physical functioning, cognitive functioning, depression symptomatology, and social activity?

2. To what extent do genetic and environmental factors contribute to initial status and change in physical functioning, cognitive functioning, and depression symptomatology?

3. How are the genetic and environmental contributions to initial status and change in physical functioning, cognitive functioning, and depression symptomatology related to social activity?

4. In monozygotic (MZ) twin pairs selected to be discordant for social activity, does the more active twin show higher levels and reduced change in physical functioning, cognitive functioning, and depression symptomatology relative to the less active twin?

\section{$\overline{\text { Method }}$ \\ Sample}

The sample is taken from participants in the LSADT, a cohort sequential study of elderly Danish twins. LSADT began in 1995 with an assessment of all members of like-sex twin pairs born in Denmark before 1920 and thus at least 75 years old at the beginning of 1995. This initial cohort was followed up in 1997, 1999, 2001, 2003 and 2005. Additional cohorts were added in 1997, 1999, and 2001 and subsequently followed at 2 -year intervals. Figure 1 gives a schematic representation of the number of participants across the multiple waves of participation. Table 1 gives some basic demographic characteristics of the sample. A total of 4731 twins have completed an intake LSADT assessment. Twins were recruited to participate regardless of whether their co-twin was alive. Consequently, many of the participants in LSADT are 'singleton twins'; the full sample includes only 1152 pairs in which both members completed an intake assessment. As can be seen, rate of participation has been excellent over the multiple waves of LSADT, with participation rates generally falling between $70 \%$ and $80 \%$. A detailed description of the LSADT sample as well as an analysis of nonparticipants can be found in Christensen et al. (1999, 2003).

The sample for the current study is based on likesex twins pairs in which both members participated in LSADT. Among the 1152 participating pairs, zygosity could not be determined for 19 pairs and 21 pairs were dropped because they were opposite-sex dizygotic twin pairs, a small number of which had been sampled in LSADT. This left a sample of 1112 twin pairs: 175 monozygotic male (MZM), 246 dizygotic male (DZM), 276 monozygotic female (MZF), and 415 dizygotic female (DZF).

Participants were usually assessed in their homes by one of approximately 100 interviewers. The interviewers were employed by the Danish National Institute of Social Research, which has extensive experience in assessing elderly Danes (Kjøller, 1996; Platz, 1989, 1990). Interviewers completed a detailed training program 2 months prior to each survey initiation and were closely monitored during the survey period. Many of the interviewers participated in multiple LSADT waves. To minimize bias, the two members of a twin pair were always assessed by different interviewers. Interviews typically lasted approximately 75 minutes and included assessment of demographic background, medical and physical health, depression symptomatology and cognitive testing.

\section{Measures}

The current study uses standard measures of physical, cognitive and emotional functioning that have been used in several previous LSADT studies as well as a measure of social activity, which is introduced in the present study.

Physical functioning. Our self-reported assessment of physical functioning is based on an adaptation of an instrument that was developed and previously validated in Denmark (Avlund et al., 1995) and has been administered essentially unchanged throughout LSADT. Here we use the Strength scale, which consists of 11 items (e.g., walk up two flights of stairs) each responded to 
LSADT

4731 have completed

Intake assessment

1904 dropped out

1455 died
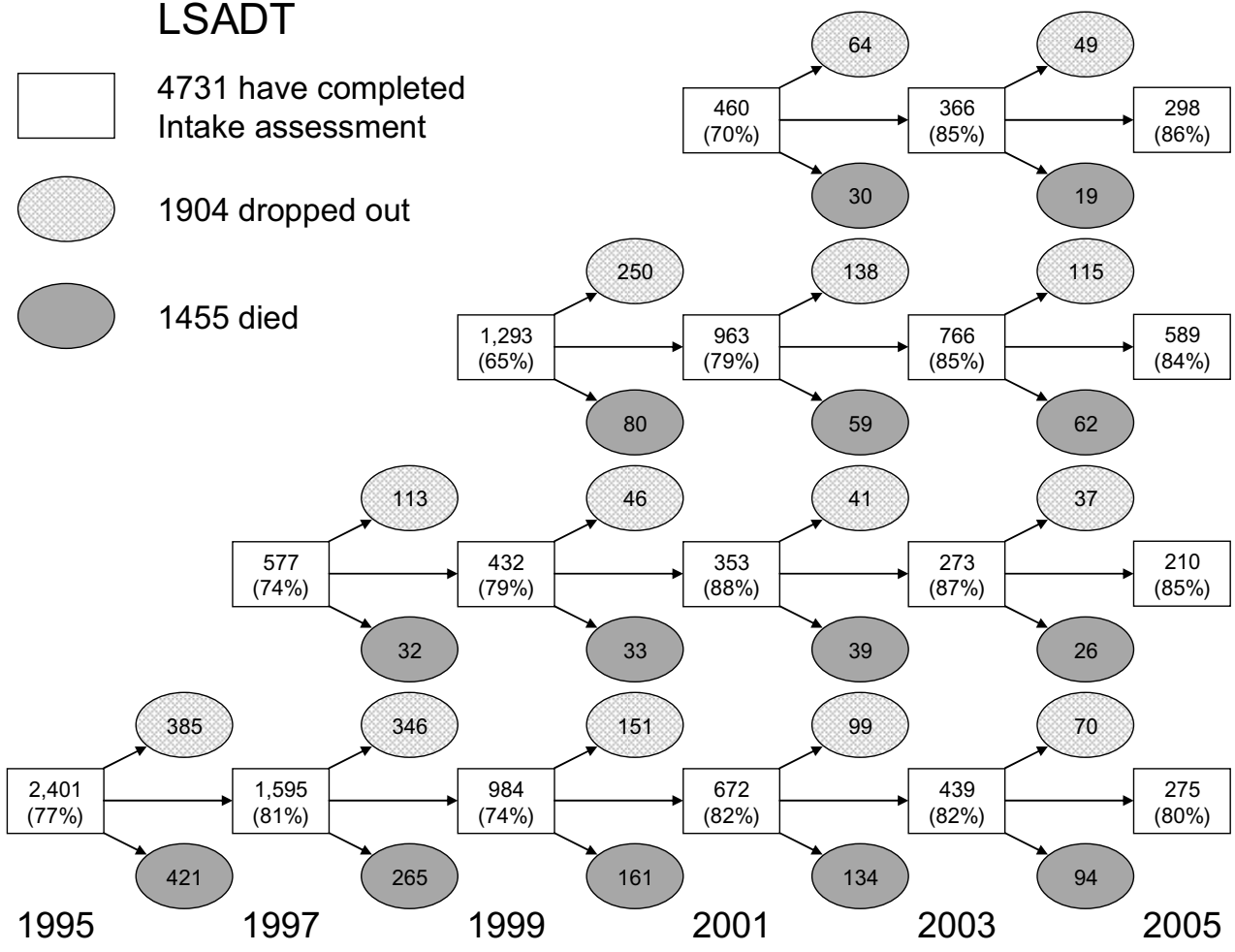

46
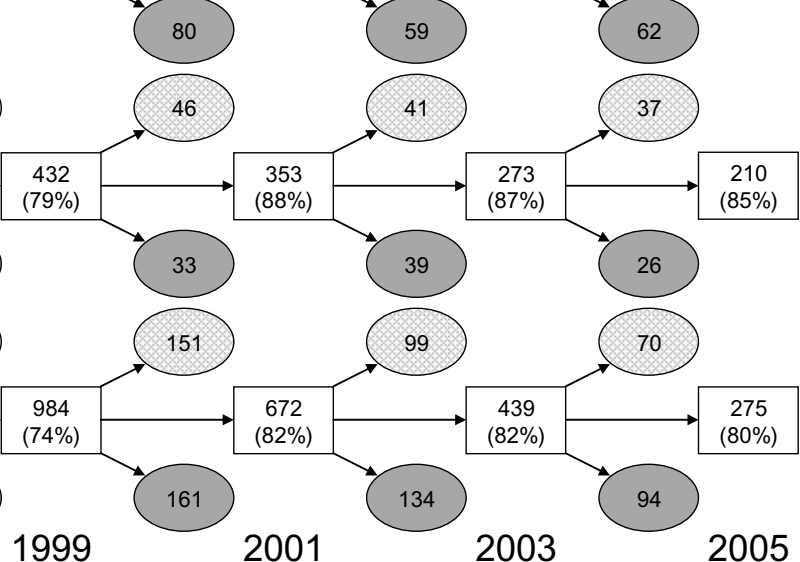

\section{Figure 1}

Participation in the multiple waves of the LSADT. The LSADT used a cohort-sequential design, with new cohorts recruited to participate at the initial wave in 1995 and at subsequent waves in 1997, 1999, and 2001 . New cohorts were not recruited after 2001 to ensure that all cohorts had completed at least three waves of assessment by the end of the study in 2005 .

on a 1 to 4 scale $(1=$ cannot do, $2=$ do with aid or major difficulties, 3 = do with fatigue or minor difficulties, 4 = do without aid or difficulty). The Strength scale is the average of the 11 individual responses. The Strength scale has high internal consistency and reliability $(>.90)$ and is relatively stable (two-year stability is generally .60 or greater).

Cognitive functioning. The cognitive battery includes the Mini Mental State Examination (MMSE; Folstein et al., 1975), and five brief cognitive tests selected to be sensitive to age-related changes (McGue \& Christensen, 2001). The MMSE is a standard neurological screen that is especially effective for screening at the low end of cognitive functioning. In our sample, the MMSE is both internally consistent (.75) and temporally stable (2-year stability $r=.64)$. The brief cognitive tests include a verbal fluency task (categories, the number of animals named in 1 minute), forward and backward digit span, and immediate and delayed recall of a 12item list. The five individual cognitive measures are

Table 1

Participants in Multiple Waves of the LSADT

\begin{tabular}{lcccccc}
\hline & \multicolumn{9}{c}{ LSADT Assessment } \\
\cline { 2 - 7 } & Intake & Follow-up & Follow-up & Follow-up & Follow-up & Follow-up \\
& & 1 & 2 & 3 & 4 & 5 \\
\hline \# individuals & 4731 & 3356 & 2401 & 1534 & 649 & 275 \\
\# twin pairs & 1152 & 703 & 459 & 247 & 79 & 18 \\
Participation rate & $73 \%$ & $81 \%$ & $81 \%$ & $85 \%$ & $85 \%$ & $84 \%$ \\
Female (\%) & $59 \%$ & $58 \%$ & $57 \%$ & $60 \%$ & $66 \%$ & $70 \%$ \\
Age Mean & 77.4 & 78.9 & 80.4 & 82.7 & 86.4 & 89.4 \\
$\quad$ SD & 5.5 & 5.1 & 4.9 & 4.5 & 3.9 & 3.4 \\
\multicolumn{1}{c}{ Range } & $70-102$ & $72-100$ & $74-100$ & $76-100$ & $81-102$ & $85-103$ \\
\hline
\end{tabular}

Note: Because of the cohort-sequential design used in LSADT not every participant was eligible to complete a third and a fourth follow-up. Participation rate is among eligible living individuals. 
temporally stable (2-year stability coefficients range from .40 to .52 ) and positively correlated, the latter justifying the formation of a composite of the five tests (which has an internal consistency reliability estimate of .75 and a 2-year stability coefficient of .60).

Depression symptomatology. Our assessment of depression symptomatology is based on an adaptation of the depression section of the Cambridge Mental Disorders of the Elderly Examination (CAMDEX; Roth et al., 1986). The Depression scale used here is a composite of responses to 17 depression items and is described more fully in McGue and Christensen (1997). The scale has high internal consistency reliability (.83) and is moderately stable over a two-year period (.64).

Social activity. The Social Activity scale is based on six items that assess the frequency with which the individual is engaged with others (e.g., how often do you leave your home, how often do you go to a party) and mental pursuits (e.g., how often to you engage in a hobby). Each item is rated on a 1 (Never) to 4 (5-7 days a week) scale. The composite scale is moderately internally consistent $(.51)$ but quite stable over a 2-year interval $(r=$ .88). Because the Social Activity scale was not administered in LSADT until 1997, all analyses reported on here are based on the 1997 and subsequent assessments.

\section{Analysis}

Four different types of analyses were undertaken to investigate the relationship of social activity with late-life functioning. First, growth curve models were fit to each of the four outcome variables as well as Social Activity. These analyses were undertaken using SAS Proc Mixed to account for the clustered structure of the data. The growth curve analyses allowed us to describe how each of the variables we are investigating was associated with age and thus provided a foundation for all subsequent analyses.

Second, biometric growth curve models were fit to each of the four outcome variables, Strength, Depression, MMSE, and the Cognitive Composite, using the methods described by Neale and McArdle (2000). In the biometric growth curve model, a schematic representation of which is given in Figure 2, the variance of an observed score at any wave is modeled as a function of an overall level effect (L), a rate-of-change or slope effect (S), and wave-specific residual effects. Using standard biometric assumptions, the slope, level and residual effects can be furthered decomposed into additive genetic (A), shared environmental $(\mathrm{C})$ and nonshared environmental (E) components. Although in principle we could model biometric contributions to quadratic and higher order moments of change given that we had up to five waves of data, we elected to include only a linear term because only a small proportion of our sample had three or four data points and it is difficult to reliably assess individual differences in higher order moments with this number of observations (Bryk \& Raudenbush, 1992).

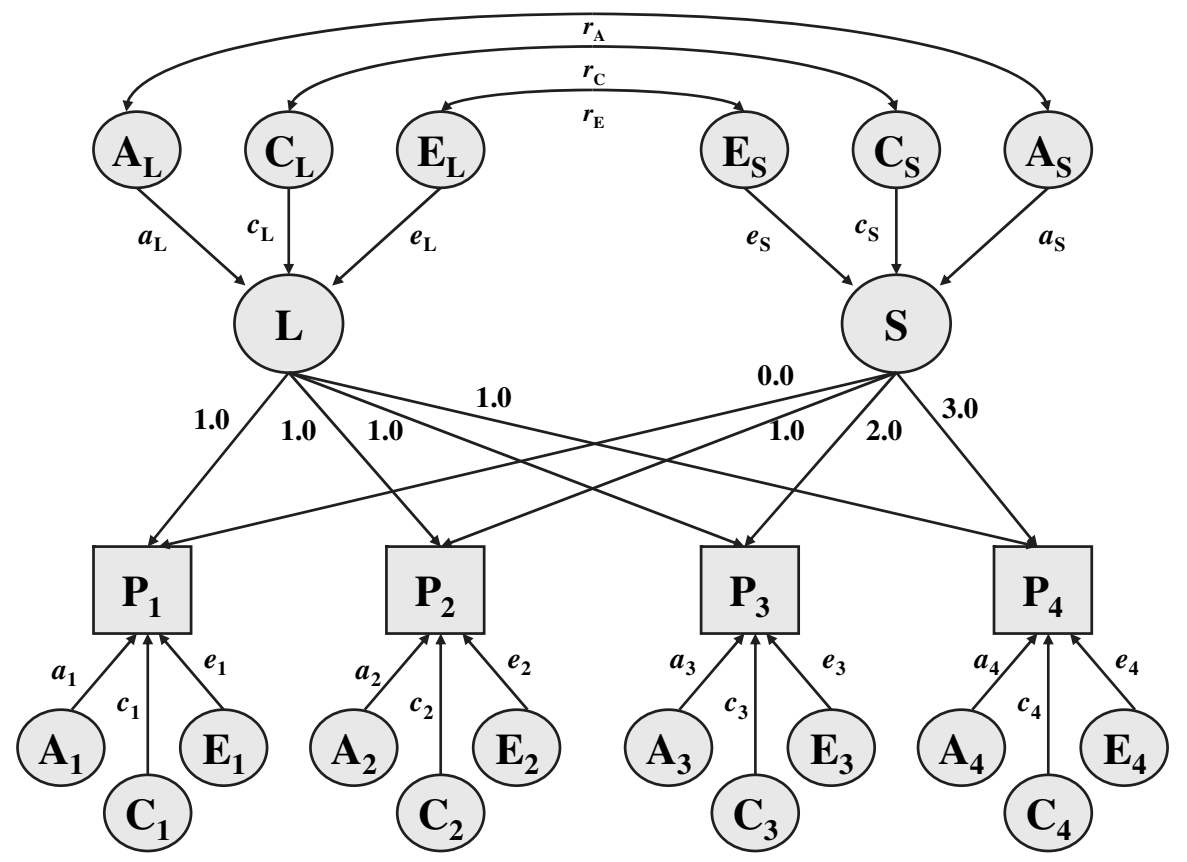

Figure 2

The biometric growth curve model. For ease of presentation, the model depicts phenotypic assessments $\left(P_{j}\right)$ on up to four waves of assessment $(i=1,4)$ rather than the full six waves in LSADT. Underlying the individual phenotypic scores at each wave is an initial value or level $(L)$ effect, a linear rate-of-change or slope (S) effect, and wave-specific residual effects. L, S and the residuals are each in turn decomposed into underlying additive genetic (A), shared environmental (C), and nonshared environmental (E) effects. 
Table 2

Standardised Variance Component Estimates (95\% Confidence Interval) from the General Biometric Growth Model Fit Separately to Each of the Four Outcome Variables

\begin{tabular}{|c|c|c|c|c|c|c|}
\hline & \multicolumn{3}{|c|}{ Intercept } & \multicolumn{3}{|c|}{ Slope } \\
\hline & $a^{2}$ & $c^{2}$ & $e^{2}$ & $a^{2}$ & $c^{2}$ & $e^{2}$ \\
\hline Strength & $.39^{*}(.16, .50)$ & $.02(.00, .19)$ & $.59^{*}(.49, .69)$ & $.14^{*}(.01, .37)$ & $.04(.00, .29)$ & $.82^{*}(.63, .93)$ \\
\hline Depression & $.50^{*}(.25, .61)$ & $.00(.00, .18)$ & $.50^{*}(.39, .62)$ & $.06(.00, .90)$ & $.25(.00, .71)$ & $.69^{*}(.08,1.00)$ \\
\hline MMSE & $.25^{*}(.01, .53)$ & $.24^{*}(.02, .47)$ & $.51^{*}(.38, .65)$ & $.12(.00, .60)$ & $.10(.00, .43)$ & $.78^{*}(.37, .95)$ \\
\hline Cognitive & $.39^{*}(.21, .60)$ & $.30^{*}(.12, .46)$ & $.31^{*}(.24, .39)$ & $.18(.00, .56)$ & $.02(.00, .41)$ & $.80^{*}(.41, .99)$ \\
\hline
\end{tabular}

Note: $a^{2}, c^{2}$, and $e^{2}$ are, respectively, estimates of the proportion of variance associated with additive genetic, shared environmental, and nonshared environmental factors.

* Parameter estimate considered significant at $p<.05$ because confidence interval does not include 0 .

Third, we fit an additional series of biometric growth models in which the initial assessment on Social Activity was included as a covariate. These models allowed us to investigate the correlation of initial level of Social Activity with initial level and change in each of the four outcomes, and to estimate the genetic and environmental contributions to these correlations. Finally as a supplement to the expanded biometric growth curve analyses, we identified a set of MZ twins discordant on initial level of Social Activity and investigated whether these twins also differed on initial level and change in each of the four outcomes.

\section{Results}

\section{Individual Growth Curve Analysis}

An individual-level growth curve model was fit to each of the five variables using SAS Proc Mixed. Independent variables included age, squared age, sex, and the interactions of sex with age and age squared. In all cases the age main effect was statistically significant at $p<.01$, and the quadratic age effect was significant for all variables except Depression. The sex main effect was significant for all variables except the two cognitive outcomes, MMSE and the Cognitive Composite, but none of the interactions of sex with age and squared age were statistically significant. Figure 3 gives the fitted group mean growth curves for females (Figure 3a) and males (Figure 3b). To facilitate interpretation, each of the five variables was scaled to have a mean of 50 and a standard deviation $(S D)$ of 10 (i.e., a $T$-score metric) in the overall sample. As is evident from the graphs, the average change in functioning over the age range spanned by LSADT (approximately age 70 to 100) is substantial for both Strength and MMSE (somewhat greater than a $3 S D$ decline) and moderate for both the Cognitive Composite (a $2 S D$ decline) and Depression (a $1.5 S D$ increase). Interestingly, Social Activity is also significantly, albeit more modestly, associated with age, declining by a little over $1 S D$ on average between age 70 and 100.

\section{Biometric Growth Curve Analysis}

The biometric growth curve model described by Neale and McArdle (2000) and depicted in Figure 2 was fit to each of the four outcome variables using the $\mathrm{Mx}$ software system (Neale et al., 1999). To account for the cohort-sequential nature of the LSADT design, which resulted in a varying number of observations on study participants, models were fit to the raw data under the assumption that data were missing at random (Little \& Rubin, 1989). Only the full growth model (i.e., A, C and $\mathrm{E}$ effects on level, slope and the residuals) was fit for each variable. Submodels were not fit because in this initial biometric analysis we wanted to assess the contribution of all potential

a
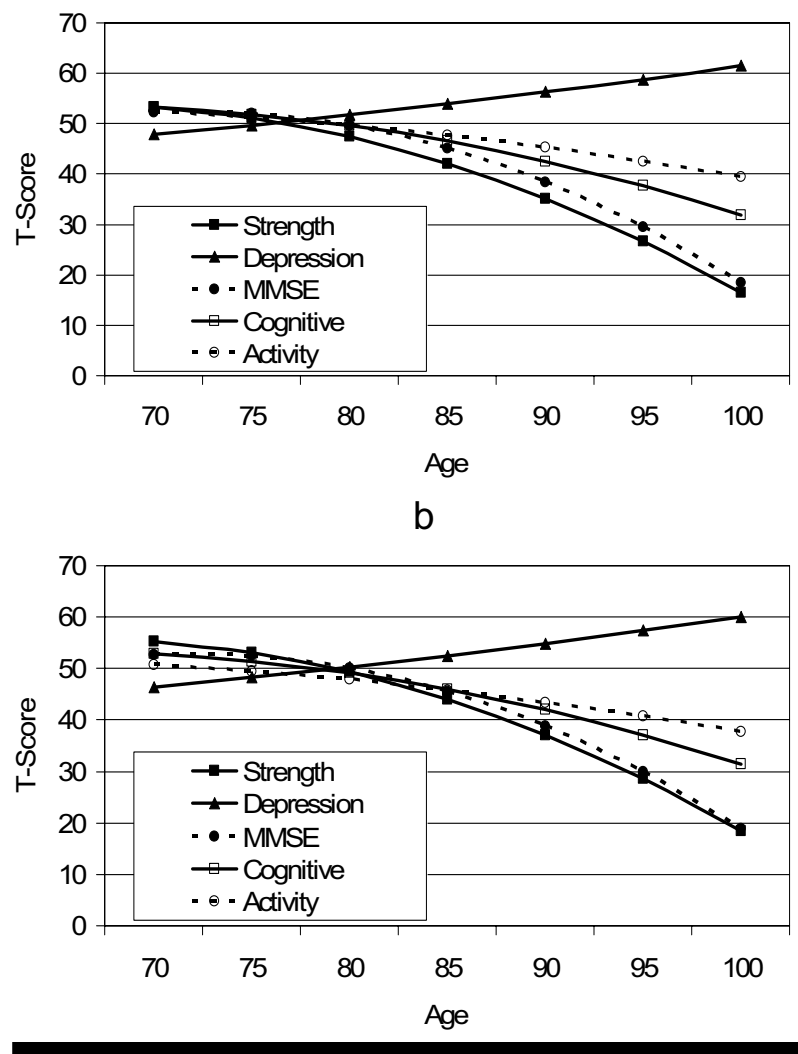

Figure 3

Group average growth curves for females (a) and males (b). Scores for each outcome have been scaled to a $T$-score metric (i.e., mean of 50 and standard deviation of 10 ) in the overall sample to ease interpretation of the magnitude of the age effects. 
a
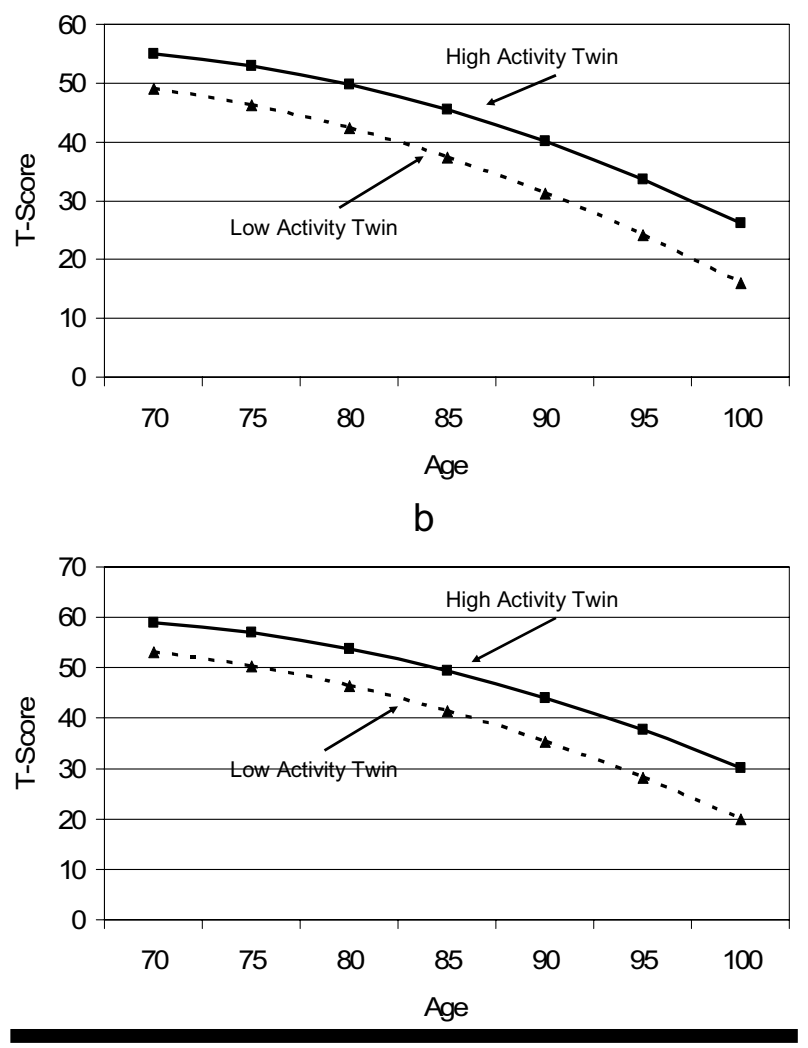

Figure 4

Discordant MZ twin growth curve for Strength for females (a) and males (b). Scores for each outcome have been scaled to a $T$-score metric (i.e., mean of 50 and standard deviation of 10 ) in the overall sample to ease interpretation of the magnitude of the age effects. The High Activity twin scored at least 1.5 standard deviations higher than the low activity twin on our measure of Social Activity. There is a significant twin difference in initial value but not change. The discordant twin growth curves for the other outcomes had a similar form to the one depicted here.

sources of variance, recognizing that the statistical power for some of the parameters in the model may be limited (e.g., the contribution of shared environmental effects, cf. Martin et al., 1978). As an alternative to formal hypothesis testing, we provide confidence intervals for the standardized variance component estimates in Table 2. For all four outcomes, the genetic contribution to individual differences in initial value (i.e., the intercept) was moderate and statistically significant (i.e., as judged by the confidence interval not including 0 ). Estimates of shared environmental effects on initial level of functioning were effectively zero for Strength and Depression but moderate and statistically significant for both of the cognitive measures. Nonshared environmental effects accounted for at least half of the variance in initial level of functioning for all outcomes except the Cognitive Composite, where it accounted for approximately a third of the variance.

The pattern of standardized variance component estimates for the slope (change) scores is quite different from that for the intercept scores. Heritability estimates are uniformly small, and in only one case, for Strength, was the estimate statistically significant. Similarly, there is little evidence for shared environmental contributions to change in functioning - none of the shared environmental variance estimates was statistically significant. The major source of individual differences in change is nonshared environmental factors, which accounted for between $69 \%$ (Depression) and $82 \%$ (Strength) of the variance in change.

\section{Relationship of Social Activity to Late-Life Functioning}

The biometric growth model depicted in Figure 2 was expanded to include intake Social Activity as a covariate and refit to each of the four outcome variables. The aim of these analyses was to estimate the contribution of Social Activity to both initial level and change in each of the four outcome variables and to determine the extent to which the association between Social Activity and late-life functioning was attributable to common genetic and common environmental factors. Before fitting these models, the standard univariate ACE model was fit to the intake Social Activity scores. The standardized variance component estimates $(95 \%$ confidence intervals) from this model were: $a^{2}=.36(.21$, $.44) ; c^{2}=.00(.00, .11)$; and $e^{2}=.64(.56, .72)$. Because the shared environmental contribution to variance in Social Activity was estimated to be 0.0 , this parameter was fixed to 0.0 when fitting the extended growth models. Shared environmental parameters were also fixed to 0.0 for Strength and Depression, since estimates of these parameters were near zero in the initial growth model analyses, but not fixed to 0.0 for MMSE and the Cognitive Composite, where the initial growth curve analyses provided evidence for shared environmental effects.

Parameter estimates describing the association of Social Activity with late-life functioning along with their associated confidence intervals are given in Table 3. At the phenotypic level, the correlation between activity and initial level of functioning was in every case statistically significant. The magnitude of this correlation in absolute value ranged from .21 for MMSE to .53 for Strength. Thus, individuals who were highly engaged socially were physically and cognitively more able and less likely to experience symptoms of depression than individuals with low levels of social engagement. Social Activity was, however, only modestly associated with change in functioning. The phenotypic correlation between Social Activity and the slope was near-zero and nonsignificant for both of the cognitive measures, and modest $(r=.18)$ but significant for Strength. This correlation was also significant for the Depression slope $(r=.22)$, although in this case the direction of correlation is counterintuitive, with rate of increase in depression being greatest among the most socially engaged individuals.

Also given in the table is the proportion of each phenotypic correlation attributable to genetic factors. Since we did not include common shared environmental effects in the model because the shared environmental 
Relationship between Social Activity and Late-Life Functioning Estimated from the Biometric Growth Model

\begin{tabular}{lcccc}
\hline & \multicolumn{3}{c}{ Biometric parameter estimate $(95 \%$ confidence interval) } \\
\cline { 2 - 5 } & \multicolumn{3}{c}{ Intercept } \\
\cline { 2 - 5 } & $\begin{array}{c}\text { Phenotypic correlation with } \\
\text { social activity }\end{array}$ & $\begin{array}{c}\text { Proportion of correlation } \\
\text { due to genetics }\end{array}$ & $\begin{array}{c}\text { Phenotypic correlation with } \\
\text { social activity }\end{array}$ & $\begin{array}{c}\text { Proportion of correlation } \\
\text { due to genetics }\end{array}$ \\
\hline Strength & $.53(.49, .56)$ & $.50(.37, .62)$ & $.18(.09, .27)$ & $1.00^{*}$ \\
Depression & $-.38(.34, .43)$ & $.63(.45, .80)$ & $.22(.09, .35)$ & $.73(.00,1.00)$ \\
MMSE & $.21(.06, .30)$ & $.74(.60, .88)$ & $.03(.00, .33)$ & $1.00^{*}$ \\
Cognitive & $.44(.40, .48)$ & $.78(.65, .89)$ & $-.02(-.15, .11)$ & $1.00^{*}$ \\
\hline
\end{tabular}

Note: "In full model nonshared environmental contribution to correlation was small, nonsignificant and opposite in sign to the phenotypic correlation. In these cases, estimates were obtained from a model where the nonshared environmental contribution was fixed to 0.0 , in which case genetic factors will account for all of the phenotypic correlation and a confidence interval cannot be calculated.

effect was estimated to be zero in our univariate analysis of the Social Activity scores, the proportion of the phenotypic correlation attributable to nonshared environmental factors is simply the complement of the proportion attributed to genetic factors. Genetic factors accounted for a majority of the correlation between Social Activity and each of the intercepts and essentially accounted for the entire correlation between Social Activity and each of the slopes.

\section{Co-Twin Control Analysis of Social Activity}

Our final analysis involved a discordant MZ twin analysis of the relationship between Social Activity and the four late-life functioning measures. Among the $356 \mathrm{MZ}$ twin pairs where both members had completed the Social Activity scale at intake, we identified those whose Social Activity scores differed by at least 1.5 SDs. While the decision to use a threshold of 1.5 $S D$ s is admittedly arbitrary, it did provide a large enough sample of discordant $\mathrm{MZ}$ twins $(N=70)$ to produce statistically reliable results. The sample of discordant MZ twins was $63 \%$ female and had an average (SD) age of $75.7(5.2)$ at the time they completed the Social Activity measure. For each of the four outcomes, the discordant twin sample was analyzed using individual growth curve analysis using SAS Proc Mixed. In these analyses, the independent variables were sex, age, squared age, Social Activity level (i.e., the high vs. the low scoring twin) and the interaction of Social Activity with age. The Social Activity main effect was significant for Strength, $F(1,69)=61.1 ; p<.001$, Depression, $F(1,69)=9.5$; $p<.01$, and the Cognitive Composite, $F(1,68)=14.1$; $\mathrm{p}<.001$; but not for MMSE, $F(1,69)=.42 ; p>.25$. In no case was the interaction of Social Activity by age statistically significant (all $p$ s $>.10$ ). Figure 4 illustrates for one of the outcomes, strength, the nature of the co-twin control growth model results.

\section{Discussion}

Summary of Results

Our analysis of late-life functioning in a large longitudinal study of older Danish twins led to the following findings: (1) over the age range spanned by LSADT, we observed significant and substantial declines in physical and cognitive functioning and increases in depression symptomatology, (2) while genetic factors contributed significantly to initial levels of physical and cognitive functioning and depression symptomatology, change in these measures was attributed overwhelmingly to the effect of nonshared environmental factors, (3) our measure of social activity was in part heritable and predictive of initial level of physical and cognitive functioning and depression but was largely uncorrelated with change in late-life functioning; and (4) MZ twins discordant for level of social activity differed in initial level but not in change in physical and cognitive functioning and depression. We discuss the implications of these findings for understanding the nature of late-life functioning and the influence of social activity on that functioning.

\section{The Nature of Late-Life Functioning in Physical and Cognitive Functioning and Depression}

Our individual growth curve analyses confirm earlier research showing that on average physical functioning (Frederiksen et al., 2006) and cognitive functioning (Salthouse \& Ferrer-Caja, 2003) decline while depression symptomatology (Gatz \& Hurwicz, 1990) increases with age. Perhaps most notable in these analyses is the magnitude of the age-related change, estimated to be approximately a $3 S D$ decline in physical functioning, a $2 S D$ decline in cognitive functioning, and a 1.5 SD increase in depression symptomatology score between age 70 and 100 .

The biometric extension of the individual-growth curve analyses allowed us to estimate genetic and environmental contributions to late-life functioning and change. Consistent with previous research (Christensen et al., 2002, 1999; Finkel et al., 2003, 1995; Gatz \& Hurwicz, 1990; McGue \& Christensen, 2003; Pedersen et al., 1992), we found moderate and significant genetic effects on overall level of physical functioning $\left(a^{2}=.39\right)$, cognitive functioning $\left(a^{2}=.25\right.$ for MMSE and .39 for the Cognitive Composite), and depression symptomatology $\left(a^{2}=.50\right)$. Because the 
growth curve analysis allowed us to partial out the effects of time-specific measurement error, these heritability estimates are somewhat greater than those for similar measures but based on a single assessment in older populations. Nonetheless, the overall strength of genetic contributions to individual differences in each of the three major domains of late-life functioning we investigated is at best moderate. Clearly environmental factors play a very important role.

This conclusion gains further support from our biometric analysis of change. In every case, the heritability of change in functioning (as reflected by the slope) was estimated to be small. The estimated heritability for the slope was .12 for MMSE, .18 for the Cognitive Composite, and .06 for Depression, all nonsignificant values. Moreover, although the heritability for change in Strength was statistically significant, the estimate $\left(a^{2}=.14\right)$ was also modest. Our results are thus consistent with earlier studies (e.g., Christensen et al., 2003; Reynolds et al., 2002) in indicating that nonshared environmental factors are the major contributors to linear change in late-life functioning. Worth noting is that in the biometric growth model measurement error, which typically is apportioned to the estimate of nonshared environment, is included in the residual terms. Consequently, our finding of strong nonshared environmental effects on the slope is not attributable to the unreliability of measurement.

\section{The Role of Social Activity in Late-Life Functioning}

Epidemiologists have repeatedly shown that individuals who are socially and intellectually engaged in late life are more likely to be physically (Prohaska et al., 2006) and cognitively (Arbuckle et al., 1998; Wilson et al., 2002) fit and are less susceptible to depression (Cacioppo et al., 2006) than those who experience relatively low levels of social and intellectual stimulation. Our findings confirm the existence of these associations. Our measure of Social Activity correlated significantly and moderately with all four of our outcomes. Specifically, in growth curve analyses we found that Social Activity correlated .53 with initial value for Strength, -.38 with initial Depression, .21 with initial MMSE, and .44 with initial Cognitive Composite. Although the association of social activity with latelife functioning has often been interpreted to reflect a causal influence of the former on the latter, selection effects cannot be ruled out. That is, it is possible that the association arises because high functioning individuals are more likely to be socially and intellectually active in late life than low functioning individuals.

Our finding that Social Activity is significantly and moderately heritable $\left(a^{2}=.36\right)$ supports the possibility of selection effects. Selection effects were further implicated by our finding that the association of Social Activity with initial level of functioning was in every case primarily genetically mediated. Specifically, the proportion of the phenotypic correlation between Social Activity and initial level of functioning attributable to genetic effects ranged from .50 for Strength to .78 for the Cognitive Composite.

Two sets of analyses were undertaken in an attempt to rule out the possibility that selection effects could account entirely for the association of Social Activity and late-life functioning in our sample. First, for each outcome we determined whether initial levels of Social Activity predicted change in functioning. The correlation between Social Activity and change in cognitive functioning was near-zero for both MMSE $(r=.03)$ and the Cognitive Composite $(r=-.02)$. For Depression the correlation between Social Activity and change was opposite in sign to what would we expected (i.e., we found greatest increases in depression among those with highest initial levels of social activity), and consequently likely to be a statistical anomaly. Only for Strength did we find a significant association between Social Activity and change, although even here the correlation was only modest in magnitude $(r=.18)$. Our longitudinal analyses thus do not support a strong causal contribution of Social Activity.

Our co-twin control analysis also do not support the existence of a strong causal influence of social activity on late-life functioning. We identified 70 pairs of $\mathrm{MZ}$ twins whose Social Activity scores differed by at least $1.5 \mathrm{SDs}$. Consistent with our finding of significant correlations between Social Activity and initial levels of functioning in the growth curve analyses, the more socially active twin scored significantly higher in Strength and on the Cognitive Composite and significantly lower on Depression than the less socially active twin. The difference for MMSE was in the same direction but not statistically significant. Thus there are environmental contributions to the association between late-life functioning and social activity, although the existence of these common environmental effects does not rule out selection processes. Most critical is whether the discordant MZ twins differed in change in functioning over time. In this regard our findings are unambiguous - we found no evidence that the more socially active twin was less susceptible to age-related changes in physical functioning, cognitive functioning, and depression symptomatology than the less socially active twin.

Our failure to find evidence that social activity contributes to change in late-life functioning is somewhat surprising in light of findings from the biometric growth curve analyses. These analyses showed that nonshared environmental factors were the major source of change in the outcomes we investigated, and review of recent gerontological research would likely lead to the conclusion that social engagement is a prime candidate underlying that change. Moreover, univariate analysis of our social activity measure revealed that approximately two-thirds of the variance in this measure was associated with nonshared environmental factors. Nonetheless, social activity accounted for little of the nonshared environmental effects on initial levels of functioning and essentially 
none of the nonshared variance in change in functioning. If not social activity, then what are the factors that account for the substantial nonshared environmental influences our analyses imply exist? We favor two alternative hypotheses. First, individual differences in age-related change may be largely stochastic and idiosyncratic. In their review of the literature at earlier developmental stages, Turkheimer and Waldron (2000) concluded that there was little evidence that nonshared environmental influences on behavior were systematic, a scenario characterized as 'gloomy' since it implies that major environmental influences will not be identified because they do not exist (Plomin \& Daniels, 1987). Second, our failure may stem from the nature of our environmental assessment. Our social activity measure was based on a single assessment in late life. An understanding of environmental contributions to late-life functioning may, however, require a broader developmental perspective. In particular, since the habits and attitudes established earlier in life may have profound effects on late-life functioning, we hypothesize that experiences in mid-life may be more critical to understanding aging than those in late life. Although it provides only weak support, our finding that social activity effects are associated primarily with initial level of functioning is consistent with this hypothesis.

\section{Limitations}

The results reported herein should be interpreted in the context of three research limitations. First, our measure of Social Activity consists of only six items, and could not possibly therefore cover the full range of social and intellectual experience that exists in late life. We also did not assess social support or positive social engagement, which may be key to understanding social effects on late-life functioning. Better measures than ours could yield different results. Nonetheless, it is perhaps worth noting that despite its limitations our measure of social activity was significantly related to initial level of functioning in all the domains investigated; it was just not associated with change in either the growth curve or discordant twin pair analyses. Second, our sample is based in Denmark, a small and relatively ethnically homogeneous country. The nature of aging in a country like Denmark, where access to medical care is universal and poverty rates are low, may differ from that in other regions of the world. Third, the nature of social activity may be different for twins than for nontwins because a twin has someone he or she has shared a lifetime with. While we acknowledge that twin research on social activity may not fully generalize to nontwins, we do note that there is no evidence that twins age differently or have different mortality rates than nontwins (Christensen et al., 1995). Finally, in our biometric growth curve analyses we defined the phenotypes by the wave of assessment (e.g., intake, first follow-up) rather than the age at assessment (e.g., functioning at age 77, 79). The latter, age-basis approach, would have accounted for age differences among participants at any given assessment. Failure to take age into account may have resulted in an overestimation of shared environmental effects (McGue \& Bouchard, 1984).

\section{Conclusion}

Analysis of a large longitudinal sample of older Danish twins showed that while social activity was significantly associated with initial levels of physical functioning, cognitive functioning, and depression symptomatology, it did not predict change in these variables. Moreover, an analysis of $\mathrm{MZ}$ twins discordant for level of social activity found that the highly active twin was not less susceptible to age-related declines in physical functioning and cognitive functioning and increases in depression symptomatology. Consequently, these analyses suggest that the association of social activity and late-life functioning largely reflect selection effects rather than true causation. Nonetheless, the major contributor to change in latelife functioning is nonshared environmental factors, the specific nature of which remains to be determined.

\section{Acknowledgments}

This work was supported by grants from the U.S. National Institute on Aging (P01-AG08761) and the Danish National Research Foundation.

The authors acknowledge funding support from the National Institute on Aging. The views expressed are those of the authors of this paper, and not the National Institute on Aging, the National Institutes of Health or the Department of Health and Human Services.

\section{References}

Arbuckle, T. Y., Maag, U., Pushkar, D., \& Chaikelson, J. S. (1998). Individual differences in trajectory of intellectual development over 45 years of adulthood. Psychology and Aging, 13, 663-675.

Avlund, K., Davidesn, M., \& Schultz-Larsen, K. (1995). Changes in functional ability from ages 70 to 75 : A Danish longitudinal study. Journal of Aging and Health, 7, 254-282.

Bryk, A. S., \& Raudenbush, S. W. (1992). Hierarchical linear models: Applications and data analysis methods. Newbury Park, CA: Sage.

Cacioppo, J. T., Hughes, M. E., Waite, L. J., Hawkley, L. C., \& Thisted, R. A. (2006). Loneliness as a specific risk factor for depressive symptoms: Cross-sectional and longitudinal analyses. Psychology and Aging, 21, 140-151.

Christensen, K., Frederiksen, H., Vaupel, J. W., \& McGue, M. (2003). Age trajectories of genetic variance in physical functioning: A longitudinal study of Danish twins aged 70 years and older. Behavior Genetics, 33, 125-135.

Christensen, K., Gaist, D., Vaupel, J. W., \& McGue, M. (2002). The genetic contribution to rate-of-change in functional abilities among Danish twins aged 75 years 
and older. American Journal of Epidemiology, 155, 132-139.

Christensen, K., Holm, N. V., McGue, M., Corder, L., \& Vaupel, J. W. (1999). A Danish population-based twin study on general health in the elderly. Journal of Aging and Health, 11, 49-64.

Christensen, K., Vaupel, J. W., Holm, N. V., \& Yashin, A. I. (1995). Mortality among twins after age-6: Fetal origins hypothesis versus twin method. British Medical Journal, 310, 432-436.

Crowe, M., Andel, R., Pedersen, N., Johansson, B., \& Gatz, M. (2003). Does participation in leisure activities lead to reduced risk of Alzheimer's disease? A prospective study of Swedish twins. Journals of Gerontology. Series B: Psychological Sciences, 58, P249-P255.

Finkel, D., Pedersen, N., Reynolds, C. A., Berg, S., de Faire, U., \& Svartengren, M. (2003). Genetic and environmental influences on decline in biobehavioral markers of aging. Behavior Genetics, 33, 107-123.

Finkel, D., Pedersen, N. L., McGue, M., \& McClearn, G. E. (1995). Heritability of cognitive abilities in adult twins: Comparison of Minnesota and Swedish data. Behavior Genetics, 25, 421-431.

Folstein, M. F., Folstein, S. E., \& McHugh, P. R. (1975). 'Mini-mental state': A practical method for grading the cognitive state of pateints for the clinician. Journal of Psychiatry Research, 12, 189-198.

Frederiksen, H., \& Christensen, K. (2003). The influence of genetic factors on physical functioning and exercise in second half of life. Scandinavian Journal of Medicine and Science in Sports, 13, 9-18.

Frederiksen, H., Hjelmborg, J., Mortensen, J., McGue, M., Vaupel, J. W., \& Christensen, K. (2006). Age trajectories of grip strength: Cross-sectional and longitudinal data among 8342 Danes aged 46 to 102 . Annals of Epidemiology, 16, 554-562.

Gatz, M., \& Hurwicz, M. L. (1990). Are old people more depressed? Cross-sectional data on the Center for Epidemiological Studies Depression factors. Psychology and Aging, 5, 284-290.

Gatz, M., Svedberg, P., Pedersen, N. L., Mortimer, J. A., Berg, S., \& Johansson, B. (2001). Education and risk of Alzheimer's Disease: Findings from the Study of Dementia in Swedsh Twins. Journals of Gerontology. Series B: Psychological Sciences and Social Sciences, 56, P292-P300.

Kjøller, M. (1996). Health and morbidity in Denmark, 1994. Copenhagen: Danish Institute for Clinical Epidemiology.

Kujala, U. M., Kaprio, J., \& Koskenvuo, M. (2002). Modifiable risk factors as predictors of all-cuase mortality: The roles of genetics and childhood environment. American Journal of Epidemiology, 156, 985-993.

Kujala, U. M., Kaprio, J., Sarna, S., \& Koskenvuo, M. (1998). Relationship of leisure-time physical activity and mortality: The Finnish Twin Cohort. Journal of the American Medical Association, 279, 440-444.

Little, R. J. A., \& Rubin, D. B. (1989). The analysis of social science data with missing values. Sociological Methods and Research, 18, 292-326.

Martin, N. G., Eaves, L. J., Kearsey, M. J., \& Davies, P. (1978). Power of classical twin study. Heredity, 40, 97-116.

McGue, M., \& Bouchard, T. J. (1984). Adjustment of twin data for the effects of age and sex. Behavior Genetics, 14, 325-343.

McGue, M., \& Christensen, K. (1997). Genetic and environmental contributions to depression symptomatology: Evidence from Danish twins 75 years of age and older. Journal of Abnormal Psychology, 106, 439-448.

McGue, M., \& Christensen, K. (2001). The heritability of cognitive functioning in very old adults: Evidence from Danish twins aged 75 years and older. Psychology and Aging, 16, 272-280.

McGue, M., \& Christensen, K. (2003). The heritability of depression symptoms in elderly Danish twins: Occasion-specific versus general effects. Behavior Genetics, 33, 83-93.

Mortimer, J. A. (1997). Brain reserve and the clinical expression of Alzheimer's disease. Geriatrics, 52(Suppl. 2), S50-S53.

National Institute of Health (NIH) Consensus Panel on Physical Activity and Cardiovascular Health. (1996). Physical activity and cardiovascular health. Journal of the American Medical Association, 276, 241-246.

Neale, M. C., Boker, S. M., Xie, G., \& Maes, H. H. (1999). Mx: Statistical modeling (5th ed.). Richmond, VA: Department of Psychiatry.

Neale, M. C., \& McArdle, J. J. (2000). Structured latent growth curves for twin data. Twin Research, 3, 165-177.

Pedersen, N. L., Plomin, R., Nesselroade, J. R., \& McClearn, G. E. (1992). A quantitative genetic analysis of cognitive abilities during the second half of the life span. Psychological Science, 3, 346-353.

Platz, M. (1989). The elderly in their homes: Vol. 1. Living conditions. Copenhagen: The Danish National Institute of Social Research.

Platz, M. (1990). The elderly in their homes: Vol. 2. How do they cope? Copenhagen: The Danish National Institute of Social Research.

Plomin, R., \& Daniels, D. (1987). Why are children in the same family so different from one another? Behavior and Brain Sciences, 10, 1-60.

Prohaska, T., Belansky, E., Belza, B., Buchner, D., Marshall, V., McTigue, K., Satariano, W., \& Wilcox, S. (2006). Physical activity, public health, and aging: Critical issues and research priorities. Journals of Gerontology. Series B: Psychological Sciences and Social Sciences, 61, S267-S273. 
Reynolds, C. A., Finkel, D., Gatz, M., \& Pedersen, N. L. (2002). Sources of influence on rate of cognitive change over time in Swedish twins: An application of latent growth models. Experimental Aging Research, 28, 407-433.

Roth, M., Tym, E., Mountjoy, C. Q., Huppert, F. A., Hendrie, F. A., Verma, S., \& Goddard, R. (1986). CAMDEX: A standardised instrument for the diagnosis of mental disorder in the elderly with special reference to the early detection of dementia. British Journal of Psychiatry, 149, 698-709.

Rowe, J. W., \& Kahn, R. L. (1999). Successful Aging. New York: Dell.

Salthouse, T. A., \& Ferrer-Caja, E. (2003). What needs to be explained to account for age-related effects on mul- tiple cognitive variables? Psychology and Aging, 18, 91-110.

Turkheimer, E., \& Waldron, M. (2000). Nonshared environment: A theoretical, methodological, and quantitative review. Psychological Bulletin, 126, 78-108.

Vaillant, G. E., \& Mukamal, K. (2001). Successful aging. American Journal of Psychiatry, 158, 839-847.

Wilson, R., Mendes de Leon, C., Barnes, L., Schneider, J., Bienias, J., Evans, D., \& Bennett, D. A. (2002). Participation in cognitively stimulating activities and risk of incident Alzheimer disease. Journal of the American Medical Association, 287, 742-748. 